\title{
Dissociation of movement initiation without dissociation of response choice'
}

DALBIR BINORA AND HELMUT REICHERT

MCGILL UNIVERSITY
The chemical (drug) state of 24 rats was changed between training and test performances in a T-maze discrimination task. This did not affect response choice (proportion of correct turns), or response execution (running time), but retarded movement initiation(start time). Thus the processes involved in response choice and movement initiation are not identical, and the movement initiation processes are the more susceptible to changes in S's chemical state.

Dissociation here refers to the lack of transfer of a trained response from one organismic (e.g., chemical) state to another. Overton (1964) found that a positional $\mathrm{T}$-maze escape response trained in a (subanesthetic) barbiturate state failed to appear when $\mathrm{S}$ was tested in the normal, nondrug, state; nor was there any transfer to the drug state after training in the normal state. This suggests that the processes involved in correct response choice are impaired when S's drug state is altered. Employing nondiscriminative responses, Bindra, Nyman \& Wise (1965) found that barbiturate-induced dissociation occurred only when the test performance required active movement; performance requiring immobility or the withholding of a response transferred well between the barbiturate and nondrug states. Bindra, Nyman, and Wise suggested the possibility that response choice and movement initiation involve separate processes and that they are subject to dissociation to different degrees.

One direct test of the above suggestion would consist in determining whether dissociation of movement initiation can occur without dissociation of response choice in one and the same experimental arrangement. The present experiment was aimed at answering this question.

Method

Twenty-four adult male rats were divided into two equal groups, a group trained in a phenobarbital state and tested in the nondrug state (PhS-NDS group) and a group for which these treatments were reversed (NDS-PhS group).

A wooden T-maze, with an electrifiable grid-floor was used for avoidance training. It was 6 in. wide and $4 \mathrm{in}$. high. Its center alley was $17 \mathrm{in.}$ long and each of the two goal arms was $19 \mathrm{in.}$ long. The initial $7 \mathrm{in.}$ of the center alley served as a start box and the terminal 11 in. of each goal arm served as a goal box. Cue cards, consisting of vertical or horizontal .5-in.wide black and white stripes, could be attached to the entrance door to each goal box. An electric shock generator-scrambler was connected to the grid-floor and set to deliver a shock of $.8 \mathrm{ma}$. The two goal boxes were made safe from shock by placing wooden platforms on the floor.

A photo-cell in the center alley, placed 6 in. from the start gate, was employed to measure response latency (i.e., the time elapsing between the raising of the start gate and the rat's passage across the photo-cell) and response time (i.e., time elapsing between the rat's passage across the photo-cell and its entry into the goal box).

The PhS was produced, as required, by an intraperitoneal injection of $30 \mathrm{mg} / \mathrm{kg}$ of sodium phenobarbital, injected $15 \mathrm{~min}$. before the daily trials.

After preliminary escape training, each $\mathrm{S}$ was trained on a schedule of one 10-trial session per day; the intertrial interval was about $1 \mathrm{~min}$. The rat was placed in the start box facing backwards. About 2 sec. later the door was opened and simultaneously a 10-sec. delay timer was turned on. If $S$ had not reached the open (correct) goal box within 10 sec., the shock was automatically turned on and it remained on until the response was completed. The cue cards were alternated from side to side according to the following two-day sequence, LRRLLRLRRL, RLLRRLRLLR. An error was recorded if the rat took the wrong turn and tried to push the (locked) door into the wrong goal box; the correction procedure was followed. For half the animals in each group the vertical stripes signified the open goal box, for the other half horizontal. The experiment was run on a schedule of six days per week.

When the rat reached the criterion of a minimum of eight avoidance responses in one session, the procedure was slightly altered. If the rat started the response (i.e., broke the photo-cell beam) but failed to complete the response within $10 \mathrm{sec}$., it was given only momentary "prompting" shocks whenever it became stationary until the response was completed. However, if the rat did not start the response within 10 sec. shock was applied throughout the remainder of the trial. This procedure was designed to minimize exposure to shock while insuring that a response choice would be made on every trial.

When an animal reached the criterion of a minimum of nine correct choices in one session (whether or not they were successful avoidance responses) on three consecutive training days, test sessions were begun. Test sessions were given in the "other" drug state, but apart from this the testing procedure was the same as in the later part of the training. 


\section{Results}

Both groups, PhS-NDS and NDS-PhS, reached the criterion of acquisition in about the same mean number of training sessions (8.8 and 8.9 days, respectively). In other respects (e.g., number of "prompting" shocks given) too there were no noteworthy differences between the two groups.

Figure 1 presents the data for the four training sessions immediately preceding the change in drug state and for the four test sessions. In the transition from one drug state to the other there was only a slight decrease in the number of correct responses; the mean number of correct responses remained close to 9 out of 10 . All rats clearly retained the discrimination following the change in drug state. Group means of the daily response latency (median of 10 daily trials) showed that the change in drug state resulted in a marked increase in response latency in both groups. Significance tests for correlated samples showed the increase to be significant in both groups (PhS-NDS: $t=2.62, p<.05$; NDS-PhS: $t=4.85, p<.001)$. Twentytwo out of the 24 animals increased their median response latency from the last training sessions to the first test session. The corresponding increases in response times were not significant (PhS-NDS: $t=1.38$, $\mathrm{p}>.10$; NDS-PhS: $t=1.18, \mathrm{p}>.20$ ); the rather large increase seen in the curve for the PhS-NDS group was due to an erratic score (30 sec.) of one animal only. discussion

Under the conditions of the present experiment, the change in drug state produced no dissociation effect so far as the correct response choice was concerned but clearly increased start time. This supports the hypothesis that the processes involved in response choice and movement (response) initiation are notidentical, and that

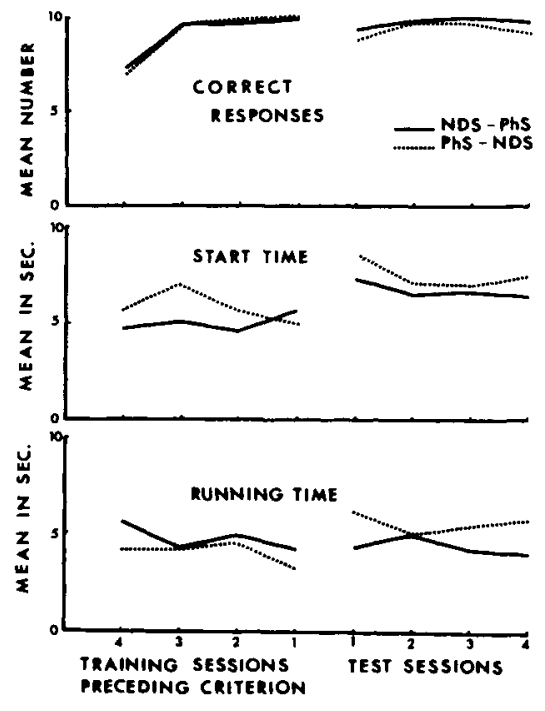

Fig. 1. Means of correct responses and start and running time scores for four pretest training sessions and four test sessions for the two groups. the movement-initiation processes are the more susceptible to changes in the drug state of the organism. The absence of an increase in running time could indicate that only movement initiation, not response execution, was impaired in the transition from one drug state to the other; but such a conclusion would have to be confirmed in an experiment without prompting shocks during test trials.

Whatever, neural impulses are required for movement initiation (see earlier discussion by Bindra, Nyman, \& Wise, 1965), these impulses can undoubtedly be generated by noxious stimulation, for no impairment of movement initiation is evident when an escape response is used. Thus, in the avoidance situation, the movement-initiation impulses must be generated by the CS, and it is this ability of the CS that must be disrupted in the transition from one drug state to the other. Recent evidence from ablation experiments (e.g., Thompson, 1964; Vanderwolf, 1963, 1964) implicates the medial thalamus, the diffuse thalamic nuclei, and the frontal cortex in the initiation of anticipatory (i.e., made in response to CS rather than US) responses. Thus a key to the understanding of the dissociation of movement initiation may lie in the change in the neural events within these structures produced by an alteration of the organism's chemical state.

The dissociation of movement initiation can apparently be demonstrated with drugs other than the barbiturates (e.g., chlorpromazine: Otis, 1964), but the dissociation of discriminative response choice has so far been demonstrated only with barbiturates or related compounds. Some minimal amount of dissociation of response choice would have been expected in the present experiment on the basis of Overton's (1964) findings. As there are many procedural differences between Overton's study and the present one, the source of the apparent discrepancy is not immediately clear.

\section{References}

Bindra, D., Nyman, K., \& Wise, J. Barbiturate-induced dissociation of acquisition and extinction: role of response initiation processes. J. comp. physiol. Psychol., 1965, 60, 223-228.

Otis, L. S. Dissociation and recovery of a response learned under the influence of chlorpromazine or saline. Science, 1964, 143, $1347-1348$.

Overton, D. A. State-dependent or "dissociated" learning produced with pentobarbital. J. comp. physiol. Psychol., 1964, 57, 3-12.

Thompson, R. A note on cortical and subcortical injuries and avoidance learning by rats. In J. M. Warren \& K. Akert (Eds.), The frontal granular cortex and behavior. New York: McGrawHill, 1964. Pp. 16-25.

Vanderwolf, C. H. The effect of medial thalamic lesions on pre viously established fear-motivated behaviour. Canad. J. Psychol., 1963, 17, 183-187.

Vanderwolf, C. H. Effect of combined medial thalamic and septal lesions on active-avoidance behavior. $J$. comp. physiol. Psychol., 1964, 58, 31-37.

\section{Note}

1. This research was supported partly by a grant (MH-03238-05) from the United States Public Health Service and partly by a grant (APT-74) from the National Research Council of Canada. 